\title{
On the shoulders of a giant
}

\author{
A long history of culture and creativity in one of the world's \\ most inspiring locales has sown the seeds for an active \\ regional innovation initiative like no other. Brett Davis \\ takes a look at the Shizuoka health science revolution.
}

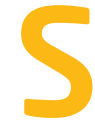
haking the lassitude of Japan's decades-long economic malaise requires resourcefulness and a new take on traditional business structures and innovation frameworks. Amidst repeated attempts by successive Japanese governments to reinvigorate the country's flagging economy, Shizuoka’s multibillion-dollar reinvention as a global technology and health leader is a demonstration of how harnessing leadership and a region's distinctive culture and traditions can pave the road to economic recovery - a modern-day industrial revolution set against the dramatic backdrop of Mount Fuji.

Cradled between the sprawling metropolises of Tokyo and Yokohama to the east, and Nagoya and Osaka to the west, Shizuoka Prefecture has for millennia played an intimate role in the culture, history and politics of Japan. With the iconic peak of Mount Fuji at its heart, the region has been home to some of the country's most ancient and influential cultures, from the tribes of the Yayoi period more than 2,000 years ago, to the first Tokugawa Shogunate in the seventeenth century. It was the collapse of Tokugawa rule in the mid-1800s that precipitated Japan's emergence from national isolation, starting the country on its eventful journey to become one of the world's great economic powers. By virtue of its location on the arterial Tokaido east-west trade route and proximity to Japan's three largest urban centres, Shizuoka has from the beginning of that economic odyssey been a crossroads where traditional Japanese values mingled with the entrepreneurial spirit of the West.

\section{Cultural centre turned} manufacturing powerhouse The Shizuoka region is traditionally renowned for green tea, mandarin oranges, wasabi horseradish and a raft of other produce that carry the tincture of the Fuji foothills. Much of that horticultural industry is still very much alive today, and the region remains Japan's largest producer of green tea and wasabi. With such fortifying local produce and an idyllic, hot spring-studded environment, it is little wonder that residents of the Shizuoka region can look forward to one of the longest life expectancies in a country renowned for its longevity. This pervasive 'Fuji spirit' of health, wellbeing and harmony with nature has coloured the region's industrial development into the modern era in some unusual ways.

The rise of Japan as one of the world's largest economies in the latter half of the twentieth century saw Shizuoka blossom

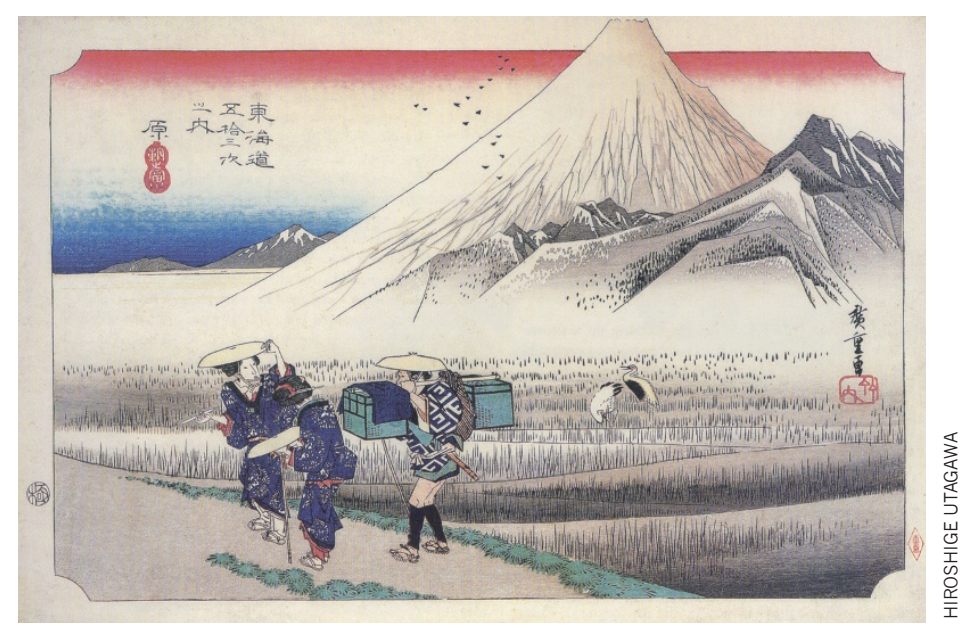

A famous 1850 s woodblock print by Utagawa Hiroshige showing travellers on the Tokaido road as it winds through the foothills of Mount Fuji in Shizuoka Prefecture.

as a manufacturing hub, coveted by business for its cheap land and location within ready freight access to Tokyo and Yokohama, Nagoya and Osaka - Japan's largest population centres. Car makers such as Honda and Suzuki were founded in Shizuoka, as were the famous piano makers Yamaha and Kawai, whose Shizuoka factories still account for the entire output of pianos from Japan. Remarkably, however, and unlike many such industrial renewals in Japan and around the world, the factories have added to, rather than displaced, the traditional resources and businesses that make Shizuoka unique - from the multitude of onsen geothermal spas that dot the picturesque Izu Peninsula, to the rolling tea plantations that comb the Fuji foothills and the bustle of fishing boats at the docks and ports along the Pacific Coast. "We are working to create a utopia," says Heita Kawakatsu, governor of Shizuoka Prefecture, "a place of good living, and good work.”

\section{A different kind \\ of innovation}

The realignment of Shizuoka's industrial focus from heavy industry such as auto parts manufacturing to health science began in the late 1990s as the prefectural government sought a solution to the accelerating exodus of companies from the region as supply contracts went to cheaper off-shore manufacturers. While many 
other areas of Japan continue to suffer from the loss of business abroad, the turnaround in Shizuoka has been impressive. In addition to boasting the largest number of companies — and particularly foreign companies - of any prefecture in Japan, those numbers are increasing year-on-year, most notably in the health-related industries. For the past several years, in fact, Shizuoka has vied for the top position in Japan in terms of manufacturing output for pharmaceuticals and medical devices.

Engineering such a monumental reversal of fortune has required a sustained effort. For decades, the prefecture has been promoting itself to foreign companies as the location of choice for business in Japan, and that proactive stance has paid off with the region topping the country in recent investments by majority foreignowned firms. The prefectural government's demonstrated commitment to the longterm support of industrial partnerships has also allowed it to secure billions of dollars in national stimulus funding.

Leading the about face is the Fuji Pharma Valley Project (PVP), a government-backed initiative aimed at leveraging the wealth of locally based health and pharmaceutical companies to establish a regional framework for collaboration on new technologies and applications in the life sciences. Central to the PVP's success is the Shizuoka Cancer Center (SCC). Set atop hills overlooking the industrial cluster in the valley below, the SCC was always core to the framework envisaged for the PVP, and has been the major beneficiary of prefectural stimulus funding. "It's part of a new type of health care complex," says SCC president Ken Yamaguchi. "We have the Shizuoka Cancer Center, which is a world-class cancer hospital and a global leader in patient-oriented care, the SCC's Research Institute with its state-of-the-art facilities and instruments, the members of the Pharma Valley Project and their depth of technological and healthcare expertise, and the Pharma Valley Center to coordinate development activity. This type of hospital-based bedside cluster for life science research and development is unique in the world, and gives industry partners the opportunity to develop solutions to needs that come directly from the bedside."

Such a patient-oriented approach to innovation in the life sciences came about as a result of the strong sense of community that permeates the Fuji region. "The Pharma Valley Project, and the Shizuoka Triangle Research Cluster initiative that encompasses Pharma Valley, Photon Valley and Food Science Hills, is based around a governmentacademia-industry partnership," says University of Shizuoka president Naohide Kinae, "but what we add — and emphasize — is the partnership with people, the citizens of Shizuoka, Japan and the rest of the world. Needs come from people."

The synergies developed among collaborating researchers, industry partners and the government lend an energized atmosphere to the innovation cluster, enticing traditional manufacturers to take on the challenge of developing medical parts and devices through close consultation with the users of their products. "We take clinical needs to local manufacturers with the technology and expertise to develop solutions," says Katsunori Ueda from the Pharma Valley Center. "We help foster local enterprise and assist businesses in negotiating the necessary regulations."

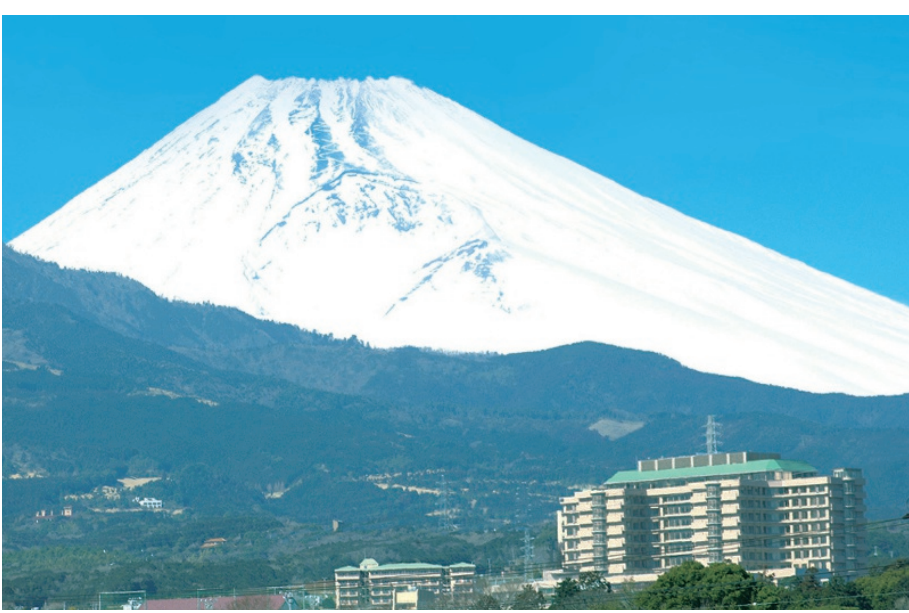

The state-of-the-art Shizuoka Cancer Center at the heart of Shizuoka's Fuji Pharma Valley Project.

\section{Exporting good health}

There is clearly something healthy about Shizuoka. Residents attribute their famed longevity to the essential role of green tea, wasabi and local seafood in their diet, and of their regular visits to the traditional onsen hot spas. Long a traditional regimen, there is now a push as part of the Pharma Valley initiatives to bring science to the region's food and lifestyle. "We call it the science of the traditional seasoning of Japan," says Kinae. "We are using the latest analytical instruments to quantify the health effects of our food, like the anti-cancer effects we believe to be associated with green tea and wasabi. Through the Food Science Hills project, we aim to export Shizuoka's good health to the world." Already next-generation green tea grown and harvested in a way to be less bitter while amplifying its health benefits is being distributed in Europe, and more products are in development.

It's not just food that is being exported, however. "Our approach at the SCC to total care for cancer patients, from the latest therapies to general health, training and the broader community care for patients or 'cancer sociology', is receiving strong interest from around the world," says Yamaguchi. The advanced platform for drug and therapy development and clinical trial network operated by the PVP's Clinical Trial Coordination Office (CTCO) is making a similarly global impact. "We are minimizing the cost to pharma for clinical trials by introducing technology such as digital patient charts and remote data access," says CTCO director Noboyuki Yamamoto. The vast library of potential drug candidates available through the PVP and the 14,000 beds of the Fuji Clinical Trial Study Network all contribute to a more convenient and streamlined therapy development process, with rare access to the bedside and the insight that provides.

In Shizuoka, a culture of ingenuity and industriousness has blended seamlessly with a distinctive Fuji spirit to foster a vibrant innovation community that has brought out the best of its industrial, academic, governmental and clinical participants. In the increasingly competitive and ultra-global business environment now faced by companies in Japan and the rest of the world alike, the 'Fuji' model is a rare success story worthy of emulation.

Brett Davis is based in the NPG Nature Asia-Pacific headquarters in Tokyo. 

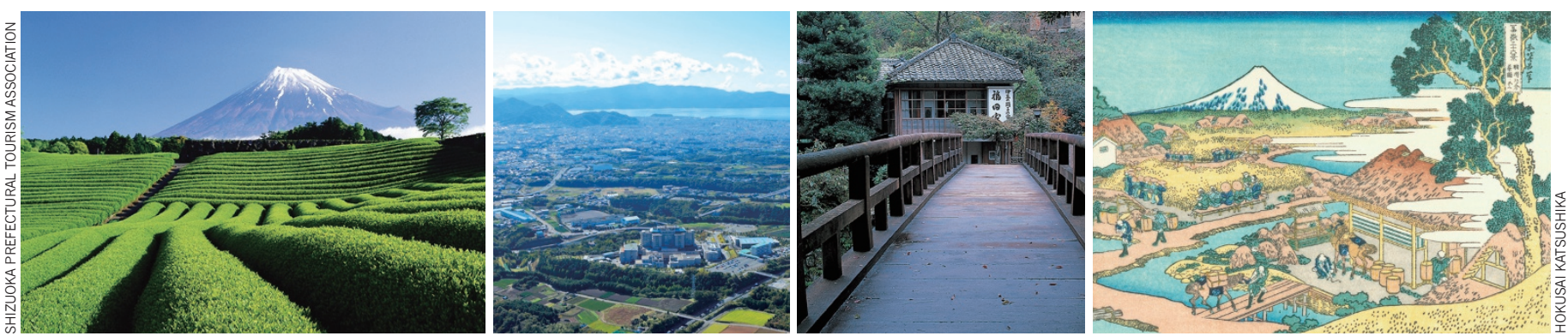

SHIZUOKA TRIANGLE RESEARCH CLUSTER

\section{Shizuoka's national treasure}

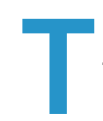

he southern foothills of Mount Fuji form the backdrop for one of Japan's most extensive innovation clusters, a concentration of local and international businesses that is reaping the benefits of cluster synergy and sustained multimillion-dollar investment under the prefectural Shizuoka Triangle Research Cluster Project.

The majestic peak of Mount Fuji is one of the world's most famous landmarks, but for the Japanese, it is an icon that embodies vastly more than a geometric marvel of geology. The mountain volcano has earned an integral place in Japan's culture and has come to symbolize the purity of nature and the underlying harmony of humanity and the natural world that are embedded in the national psyche. From its peak of 3,776 metres to the Pacific Coast just 25 kilometres to the south, the southern foothills of Mount Fuji in Shizuoka Prefecture form a rich, idyllic environment of forests, rolling hills, hot springs and exquisite scenery that has inspired artists, artisans and farmers for centuries.

"I believe that the Shizuoka region epitomizes what is distinctly Japanese," says Heita Kawakatsu, governor of Shizuoka Prefecture. "It is already well known as a good place to live and to visit; we want to show the world that it is also a remarkable place to learn and to work."

The 'Fuji' region has long held a special place in the hearts of Japanese, as both a destination for spritual healing and a source of some of the country's finest produce. Yet ever since Japan opened its borders to the wider world, Shizuoka's proximity to Tokyo and location on the main Tokaido east-west trade route has also allowed the region to benefit from an infusion of cultures of innovation and entrepreneurship from around the globe. So while the epithet 'Made in Fuji Country' might once have referred to green tea and pottery, the notion has now come to mean much more. The region is now a hive of manufacturing and pharmaceutical activity - famous internationally for its car makers Yamaha, Honda and
Suzuki - with a burgeoning innovation sector in pharmaceuticals, medical devices and healthcare products. Shizuoka's reputation as one of Japan's most active manufacturing centres is highlighted by its regular appearance as one of the top three prefectures in Japan in terms of manufacturing output for pharmaceuticals and medical devices.

What makes Shizuoka more than just a manufacturing powerhouse, however, is the concerted support of the prefectural government and an underlying 'Fuji spirit' that marries technological innovation with the needs of society and the individual. Inspired by the fertile manufacturing and pharmaceutical culture, the prefectural gov ernment launched the Shizuoka Triangle Research Cluster Project to support the establishment of local and international companies and promote innovation and collaboration in the region Shizuoka's continued prosperity is due in no small part to this innovation cluster initiative.

Forming the three 'apices' of the Shizuoka triangle are the areas known as Photon Valley in the west, Pharma Valley in the east, and Food Science Hills draping the foothills in between. Photon Valley was founded around the world-leading optics and electronics technology of companies in the city of Hamamatsu, which is also the base of the world-renowned technology company of the same name. In central Shizuoka, the Food Science Hills project aims to utilize the region's agricultural resources to produce foods with amplified health benefits or pharmaceutical enhancements.

Exemplifying the prefectural government's vision for the cluster initiative, however, is the Pharma Valley Project, centred around the Shizuoka Cancer Center in the region's east. Envisaged as a health science community with the impressive state-of-the-art Shizuoka Cancer Center at its heart, Pharma Valley is rapidly becoming a world-renowned destination for medical treatment and the development of advanced therapies and pharmaceuticals. Ten years in the planning and backed by considerable prefectural funding, the centre was established in 2002 on hills overlooking the lowlands of Pharma

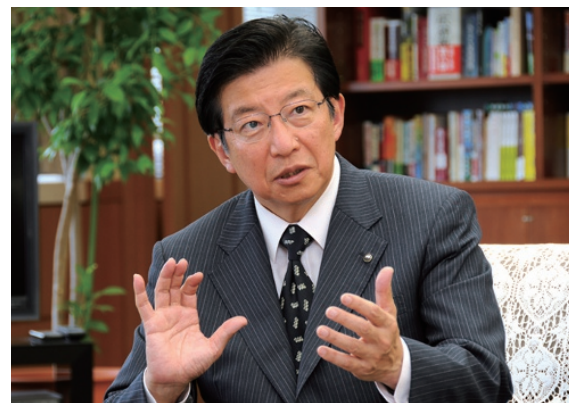

Heita Kawakatsu, Governor, Shizuoka Prefecture

Valley, and now forms an integral part of the region's healthcare and innovation infrastructure. It is one of the most advanced cancer hospitals in the world, serving as a model for cancer hospitals throughout Japan and a prestigious venue for advanced studies and specialist medicine. "The best and brightest talent from all over Japan came to join our Cancer Center," says Kawakatsu.

The Pharma Valley model, centred as it is around the Shizuoka Cancer Center, is itself unique in the world and offers some distinct advantages. "Having a hospital and bedside care as the core of a cluster for life science development means that needs for new technology arise directly from the clinic, from patient experiences," says Ken Yamaguchi, president of the Shizuoka Cancer Center. "It also facilitates translational research on new technology — the second and third generations of a device or treatment can be developed quickly here based on direct interaction with patients." So effective is the Pharma Valley model, the project has earned designation as a 'Regional Innovation Cluster Program, making it eligible for a further round of funding for research activities from the Japanese Ministry of Education, Culture, Sports, Science and Technology (MEXT).

A notable characteristic of the Pharma Valley Project is that the vast majority of innovations to arise out of its collaborations are patient-oriented, targeting the unaddressed needs of patients in care, particularly those with cancer. And the list of new products is long, from tumour markers to aid early diagnosis, to products that minimize distress in patients with advanced cancer by alleviating the distinct odour associated with the disease. 'Made in Fuji Country' still rings with nuances of healing and nurturing of the spirit - only now that succour is supported by world-class research and technological innovation. 

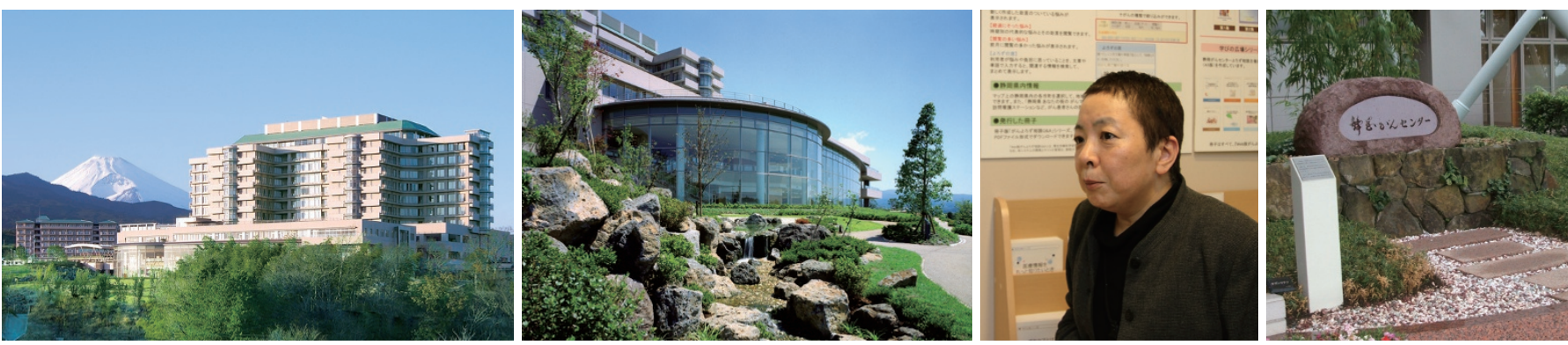

SHIZUOKA CANCER CENTER

\section{A noble cause}

\section{ituated on graceful hills overlooking Shizuoka's Pharma Valley district, the Shizuoka Cancer Center was from the outset planned and constructed as a state-of- the-art cancer hospital that unifies the very latest in cancer treatment and clinical research with a patient-focused, humanistic notion of 'harmony' that mirrors its serene location in the foothills of Mount Fuji.}

The astonishingly rapid advancement of research on the genetics and molecular biology of disease in recent years hints at a future in which many of the diseases that seem to have become more prominent in modern society, such as cancer, could be cured as a matter of routine. Amid the excitement of new technology and treatments, however, there is often something missing from this highly focused cure-based approach to medicine. "Ultimately what we do is for the patient," says Ken Yamaguchi, president and founder of the Shizuoka Cancer Center (SCC), "but a patient's experience is not limited to the surgery or the doctor's office."

This is particularly true with cancer. Every cancer experience involves a trauma that transcends the symptoms of the disease itself, and that distress extends beyond the patient to their family, friends and the community. This aspect of cancer is now recognized as 'cancer sociology'. "Every case is a narrative. Cancer affects not only the body, but also the spirit. So while we as clinicians use our expertise to treat the disease, care for the spirit should be considered just as vital. A patient's fight against cancer requires all the emotional energy they can muster, from within and from those around them. We aim to engender a 'frontier' fighting spirit in our patients, and if medicine fails to meet the challenge, a spirit of ease and acceptance knowing that we fought the good fight." This principle of total care is immediately apparent in the way the hospital is set up, with research facilities and palliative care abutting core clinical facilities. The integration of high-quality palliative care for terminal patients into the hospital proper is rare elsewhere in the world.
Now head of the SCC, the top comprehensive cancer centre in Japan and a national model for such centres throughout the country, Yamaguchi previously worked as a physician and researcher in advanced breast cancer at the National Cancer Center in Tokyo for 30 years. In that role he came to understand the profound impact cancer has on the lives of patients and their families. His experience and ability took him to the Imperial Household, where he served for many years as private physician to Princess Takamatsu and later as special advisor to other members of the royal family. In his capacity as court physician he developed a deep understanding of the royal principles of hospitality and harmony, principles that Princess Takamatsu - renowned for her support of cancer research through the Princess Takamatsu Cancer Research Fund — urged him to incorporate into clinical practice. Yamaguchi would go on to found the SCC in 2002, and the princess's inspirational words now grace a stone plaque at the centre's entrance.

"The treatment of cancer is changing rapidly, and we now have many options in addition to surgery. But treatment is just part of the equation. We need to offer total care, and in this the SCC is one of the pioneers," says Yamaguchi. Total care means just that, from physical training and nurturing of general health in the lead-up to surgery or other treatment, to 24-hour telephone support and even assistance in making special arrangements with local banks to minimize the stress of financial worries during such a difficult time

The ways in which the SCC has put total care into practice are manifold. Fundamentally, and in an intentional break from the traditionally rigid Japanese hospital hierarchy, every support provider is empowered to act in the best interest of the patient. "This is unique in Japan. We have highly trained nurses, dieticians, psychologists and physiotherapists who can provide care within their expertise as it's needed without having to wait for the primary doctor. This means that all of our staff can help in their own way, and that the patient receives support from everyone they deal with."To implement this strategy, the SCC has established

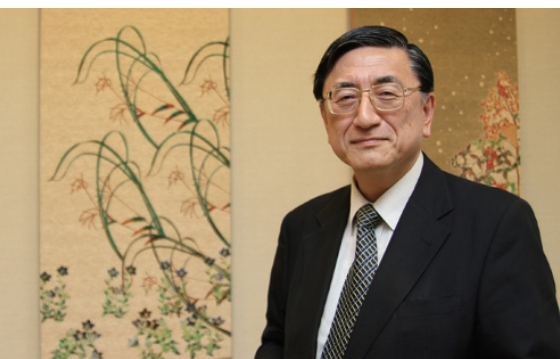

Ken Yamaguchi, President, Shizuoka Cancer Center

in-house schools for specialized nurses, the first programme of its kind to be incorporated directly into a hospital and making the centre a coveted destination for nurses from around the country.

Also unique is the SCC's Cancer Survivorship Research Division and Patient Support Center, established within the hospital as both a onestop, all-inclusive support centre and a research department analysing the anxieties and issues experienced by cancer sufferers. "Anxieties are not limited to those related to medical treatment," says division chief Mutsumi Ishikawa. "We receive enquires from SCC patients and cancer patients all over Japan. Many of the questions are related to the life of a cancer patient in the community, and many are from relatives wanting to know the best way to support a loved-one with cancer - what kind of food to prepare or how daily life can be made more relaxing." Ishikawa's division has developed a database of anxieties and burdens based on a categorization scheme developed through the analysis of almost 8,000 patient experiences. They have identified more than 10,000 anxieties that the division is working to address directly by providing information on the internet and in a series of booklets and DVDs, forming part of an 'information prescription' that is provided to every patient.

Supported by cutting-edge facilities and the latest therapies, the SCC cures more than two thousand cancer patients each year, but sadly another thousand will lose their battle with disease. "For those that survive, we want to provide a better quality of life; for those that won't, we want to provide a better quality of death," says Yamaguchi. "As human beings, we cannot help but want to ease the suffering of others. We should not stop ourselves from acting on that impulse, and in a clinical setting, that means engaging with the patient and helping them through what is a very traumatic personal experience. That is what the spirit of harmony teaches us." 

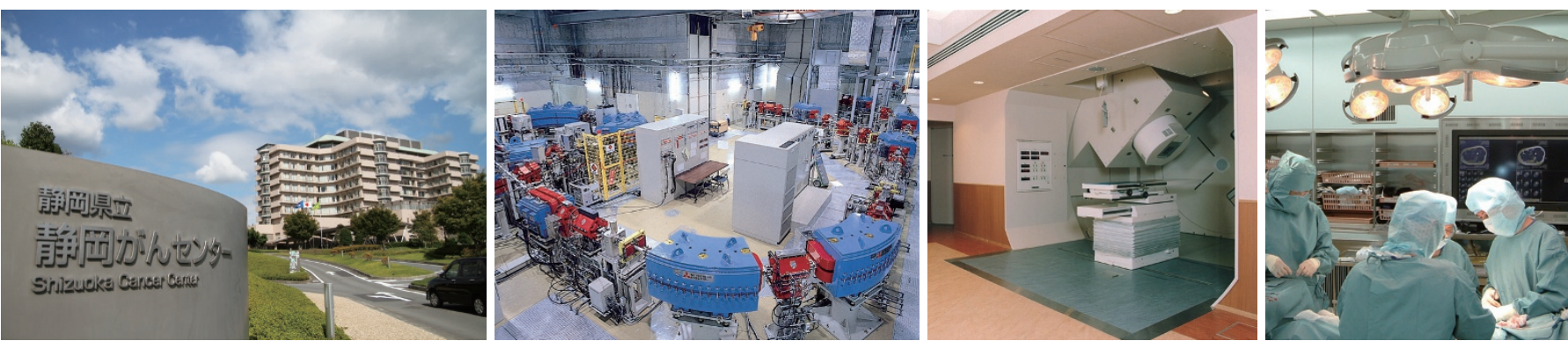

FUJI PHARMA VALLEY PROJECT

Integration breeds innovation

\begin{abstract}
1 s the beating heart of a regional innovation cluster for life science research, the Shizuoka Cancer Center provides an unparalleled framework for the development of therapies and medical devices by bringing industry to the bedside under the Fuji Pharma Valley Project.
\end{abstract}

The science underlying much of modern medicine begins in the laboratory. The road from a basic discovery by scientists in the lab to a practical clinical therapy, however, is a long, arduous and risky one. Every year billions of dollars are poured into the translational research required to take a discovery from the laboratory bench to the hospital bedside, and the increasing complexities involved in conducting such clinical research means that the costs of bringing a new therapy to market continue to rise-along with the risk of failure. And while industry is the engine-room of clinical research and development, industry-driven research is essentially one-way. A deeper and more integrated infrastructure that fosters a closer working relationship between innovators and clinicians could substantially reduce the cost and risk of therapy development while at the same time revealing development targets that better meet the needs of patients and physicians.

Supported locally by the concentration of life science companies in Pharma Valley, Ken Yamaguchi, now president of the Shizuoka Cancer Center (SCC), in collaboration with the Shizuoka prefectural government initiated the ambitious Fuji Pharma Valley Project (PVP) in 2001 with an eye to a future in which industrial and research partners can work side-by-side with doctors in the clinic to develop therapies and devices that better address the needs of patients and the community. That future was realized with the construction of the SCC and its integrated Research Institute in 2002. With total investment by the prefectural government in the PVP now exceeding one billion dollars, the project is a prime example of a successful academiagovernment-industry partnership and an implementation of a unique hospital-based bedside cluster for life science research and development.

The SCC was established as both a world leading centre for cancer treatment and a regional and international fulcrum for clinical research in close collaboration with research institutes and pharmaceutical and medical device companies. In its very construction, the SCC involved a tie-up with Mitsubishi Electric in the installation of a massive proton beam facility, one of only a score such treatment facilities around the world. The highly targeted nature of proton beam therapy makes this form of treatment particularly effective for earlystage prostate and lung cancers. "Proton beam therapy takes just 30 minutes a day, meaning that patients can visit the clinic for treatment while living at home. It also induces fewer complications compared with conventional radiation therapy, but with a much higher success rate. It is one of the ways that we try to improve the quality of life for cancer patients," says Yamaguchi. The proton beam generator itself would look more at home in a high-energy physics facility, and along with the fixed horizonta beamline and two massive rotating gantries that direct the beam with high precision, necessitated the construction of an entire dedicated hospital wing.

In keeping with its planned role as a technology leader, the hospital from the outset did away with clipboards and paper charts, developing an advanced electronic patient information system in collaboration with Fujitsu and now IBM that serves the dual roles of a paperless patient record accessible from anywhere in the hospital and a comprehensive information system for drug and therapy developers. The information system has proved no small boon for the SCC, and alongside the on-site office facilities made available to industrial and private research partners forms a major part of the attractiveness of the centre for conducting clinical trials.

The SCC has also provided a valuable proving ground for innovations that were in development before the centre's construction. A minimally invasive endoscopic treatment for stomach cancer with a remarkable 95\%-plus success rate for early stage cancers, developed by researchers at the National
Cancer Center (NCC) in Tokyo, was pioneered at the SCC and is now used routinely around the world. And a tumour marker for small-cell lung cancers, ProGRP, developed by Yamaguchi while at the NCC, has now been standardized and commercialized for sale in Europe through a joint venture between the PVP and the Chicago-based healthcare giant Abbott Laboratories.

Some of the new innovations to emerge out of the extensive network of research institutes, government agencies and pharmaceutical and medical device companies that make up the membership of the PVP are truly world-leading - innovations such as the imaging robotics system developed by Fujifilm in close collaboration with practising physicians at the SCC." The system we are developing automates the cancer diagnosis process, significantly shortening the time taken to perform diagnoses," says Yamaguchi. "We have already achieved diagnostic accuracy close to that of experienced doctors." The SCC is also developing a new diagnostic imaging system for melanoma in cooperation with Mitaka Kohki and Waseda University in Tokyo - a prestigious university that is expanding its involvement in the life sciences. "Improving our diagnosis of cancer, making more accurate diagnoses at earlier stages of disease, is something we put a lot of effort into at the SCC," says Yamaguchi, "and that means tapping the technology expertise of collaborators that may have never been involved in clinical research before."

The hospital-based bedside cluster put into practice so effectively at the SCC is unique in the world and offers an entirely new approach to clinical research. "Developers of drugs and medical devices cannot use their product themselves to treat patients," says Yamaguchi. "By inviting our industry partners into the clinic to see their products in use, they gain a better understanding of what is required to make the innovation successful, and our staff and patients can give direct feedback on possible improvements. And with all the real-time data of the advanced information system at their fingertips, our centre makes development that much easier and more effective." 

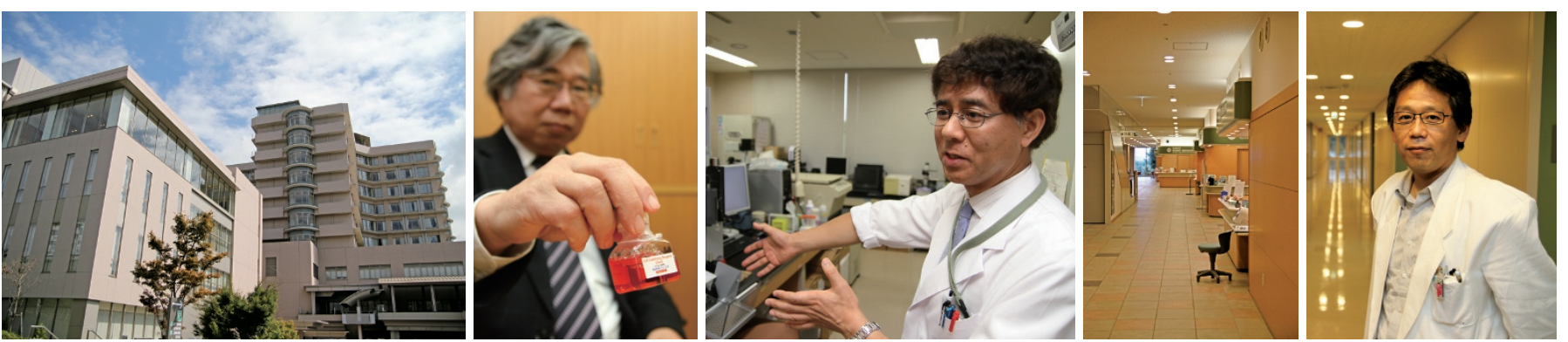

PHARMA VALLEY CENTER AND CLINICAL TRIAL COORDINATION OFFICE

A world-class platform for clinical research

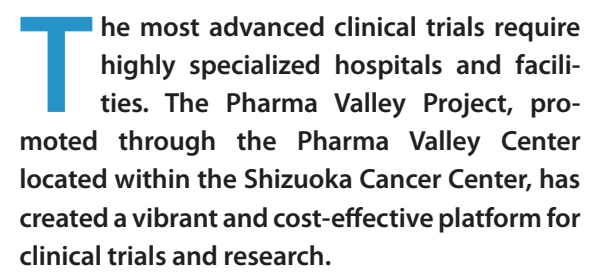

"In just eight years, we have gone from zero to a clinical trial network of 29 hospitals and 14,000 beds supporting over 200 current and scheduled trials and research investigations by companies from all over the world," says Toshiyuki Soga, director of clinical trial promotion at the Pharma Valley Center (PVC). "This has only been possible because of the support of the Shizuoka prefectural government, who took up the challenge to make Shizuoka a global exporter of healthcare and medical technology."

Soga and PVC director Ken Inoue head a motivated and highly skilled team that acts as a conduit between pharmaceutical companies, medical device manufacturers and the clinical infrastructure in which product trials and translational research takes place. "Having the best staff and administration is vital. We have highly qualified members from research institutes, government, the clinic and even the private sector. This has allowed us to develop a real public-private fusion," says Inoue.

Supported by a multimillion-dollar budget from the prefectural and national governments, the PVC and the Clinical Trial Coordination Office (CTCO) have developed a platform that offers many benefits to industry. "Our concentration of beds, and in particular our inclusion of patients in palliative care, who are often segregated out of such networks elsewhere around the world, means that we have access to a large number of suitable cases for clinical trials," says CTCO director Noboyuki Yamamoto. "So trials can be conducted faster, at lower cost and with the convenience of having many cases in one area."

The CTCO and PVC are also implementing new measures to further reduce the cost of conducting clinical trials. Already, evaluations by the independent ethics committee are provided at minimal cost to industry, but there is a much more significant cost-saving measure in the works. "The advanced patient information system developed here at the Shizuoka Cancer Center already makes source document verification much easier," says Yamamoto, "but we will soon be making the same data available remotely, which means industry will no longer need to send clinical representatives here to the clinic, a substantial cost advantage." Such remote access will be unique in the world, and with the round-the-clock service provided by the CTCO makes the Pharma Valley clinical trial platform a truly international programme. Already the platform has attracted interest from major international pharmaceutical companies such as the pharma giant AstraZeneca, which is conducting one of its Japanese clinical trials at the Shizuoka Cancer Center (SCC). "We have many phase III trials, but a characteristic of our platform is our ability to support phase I trials. We have also had very strong uptake for international and Asian trials," says Yamamoto.

With the high-quality laboratories and significant resources of the SCC and other research institutes of the Pharma Valley Project (PVP) at its disposal, the PVC oversees a comprehensive drug development platform that extends from the selection of drug targets from its library of more than 70,000 chemical compounds, through to phase III clinical trials and licensing. Niche needs documented as part of the PVC's clinical needs initiative also provide a wealth of possible targets for development. "We distribute a 'call for ideas' flier among medical staff," says Katsunori Ueda, deputy executive director of the PVC, "and then we take identified needs to industry members of the PVP to see if they can be addressed. This has resulted in some very useful niche products, and has helped promote local industry, which is one of the main goals of the PVC." Some of the devices developed so far include a de-aerating tube that markedly improves patient comfort in proton beam therapy for prostate cancer, and a series of Butler-brand oral care products by Sunstar that help with the sensitization and ulceration of the mouth during chemotherapy - both of which arose out of clinical needs and were produced through collaboration with Pharma Valley companies.

The resources of the SCC's Research Institute located adjacent to the hospital itself play a key role in supporting the research that underpins the clinical development of new therapies and drugs. Core to the scheme's success is the ability for researchers from industry to share use of the millions of dollars worth of equipment and the extensive library of potential drug targets. "There's been a fundamental shift in the way we search for and develop new drugs," says Inoue. "Whereas in the past drug effects might have been largely unknown prior to trials, the new era of molecular research throws up targets that are identified because of their known role in certain disease processes. Such research requires access to many cell and tissue samples and current disease cases, and that we can offer in abundance through the SCC's research institute."

The SCC supports a number of in-house research programmes, including an advanced cancer immunotherapy study that has already produced some remarkable results. "We have ongoing clinical trials of cell-based cancer immunotherapy for melanoma and glioma," says Yasuto Akiyama, a medical doctor now involved in immunotherapy research at the SCC. "We take monocytes from the patient, and generate a dendritic-cell-based vaccine in the laboratory that we then inject back into the patient."This experimental therapy has been trialled in 30 cases at the SCC so far with promising results, achieving a near-doubling of the survival term for advanced melanoma cases.

Akiyama is also working on biomarkers and antibody-based therapies produced by antibody gene cloning from single $B$ cells derived from the patient. "We have developed a world-leading B-cell screening and gene cloning method that reduces the vaccine generation time from months to weeks," says Akiyama. "We do everything here in-house, from 'multi-omics' to clinical application. This type of work couldn't be done without the access to patients and advanced cases possible at the SCC and the latest facilities here in the research institute." 

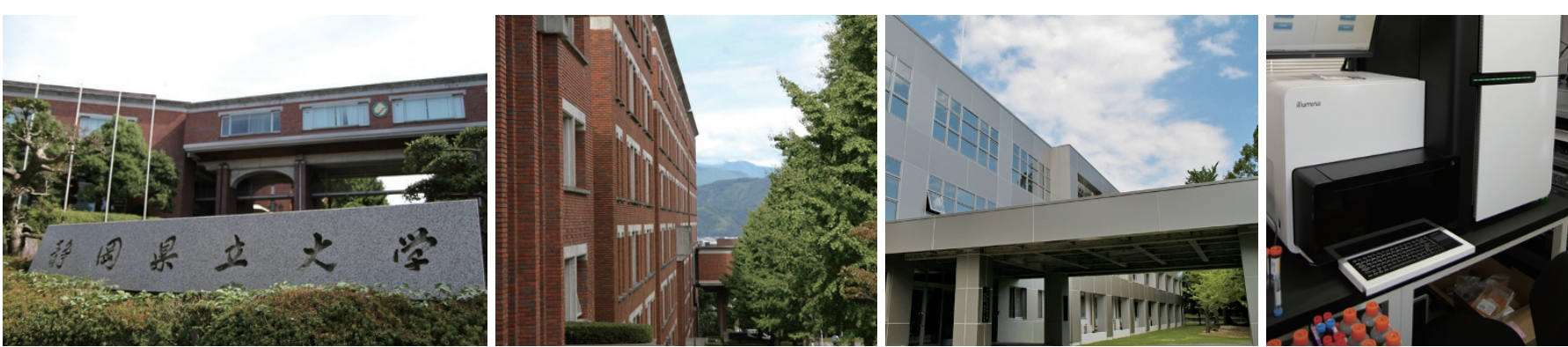

UNIVERSITY OF SHIZUOKA AND NATIONAL INSTITUTE OF GENETICS

A local destination for world-leading research

\begin{abstract}
$\mathrm{n}$ addition to being one of the most popular tourists spots in Japan, Shizuoka is also making a name for itself as a destination for researchers aiming to forge their own path in world-class life science.
\end{abstract}

The Pharma Valley Project in Shizuoka Prefecture is rightly proud of its pharmaceutical and medical device companies, but even these are not the whole story. Integral to the project's ongoing success are regional academic partners such as the University of Shizuoka and National Institute of Genetics, who produce some of the local expertise that has made the project so successful. Both have developed unique research programmes aimed at fostering the multidisciplinary research skills that are expected to become so important in the life sciences.

"Takeshi Hoshi, the second president of the University of Shizuoka, foresaw the need to establish an interdisciplinary school for nutrition research," says Naohide Kinae, current president of the University of Shizuoka. "More than twenty years later, we still have the

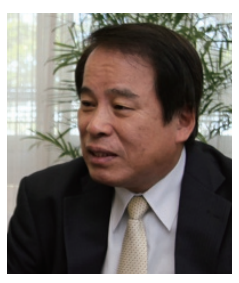

Naohide Kinae, President, University of Shizuoka only graduate school in Japan that combines food science and nutritional science under the umbrella of life science research."

Since its founding in 1987, the University of Shizuoka has always upheld a focus on the life sciences. Its schools of pharmaceutical sciences, food and nutritional sciences, and nursing form a natural fit with the needs of Pharma Valley and its extensive network of associated industries. The university's Center for Drug Discovery, in particular, has played a major role in building the expansive library of chemical compounds that form the basis for many Pharma valley ventures.

In 2002, Kinae in his capacity as head of the Graduate School of Nutrition and Environmental Sciences led the establishment of a Center of Excellence (COE) research programme aimed at investigating the interaction between food and medi- cine, such as how the efficacy of medicine can be affected by the foods we eat. "We wanted to create a new field of science centred around the role of food in medicine and health," says Kinae. "The wealth of knowledge we developed through that programme is now being applied in a new Global COE project for Innovation in Human Health Sciences."

The Global COE programme continues the systemization of this new field of study by bringing the latest technology to food analysis through collaboration with other regional research institutes and local Pharma Valley companies. "One of our major streams of study involves the use of positron emission tomography to determine the pharmacokinetics of pure and modified foods," says Kinae. "It's part of our study on food-based health and longevity, for which Japan, and particularly Shizuoka, is worldrenowned."The overall goal of the project is to construct a database of food effects and the interaction between food and medicine, with the aim of discovering biomarkers for ageing as well as developing foods and diets that prevent and even cure disease.

The National Institute of Genetics (NIG) is an inter-university research institute that focuses on the type of basic research that drives developments in the life sciences. "The NIG was established more than 60 years ago, and in that time we have seen genetics change from the study of heredity to the basis for all of life science," says Yuji Kohara, director-genera of the NIG. "Genetics is new again, and it feeds into so many aspects of molecular biology and modern clinical practice. And the remarkable diversity in biological and clinical science needs to be mirrored in the multidisciplinary knowledge of our researchers."

To accelerate the development of professionals that can contribute to life science research at the highest level, the NIG established a genetics programme as part of the Graduate University for Advanced Studies - a disseminated inter-university research initiative known as 'Sokendai' that places graduate programmes in key national research institutes. "Postgraduate research at a research institute like the NIG gives students exposure to a different kind of research culture to that found in universities", says Kohara. "It's a high-quality learning experience."
High-profile professors and the lure of real academic freedom make the NIG's graduate programme in genetics an attractive option for advanced studies in life sciences - that, and the world-class facilities and resources that support the institute's 39 laboratory programmes. "The NIG continuously upgrades its facilities for high-throughput DNA

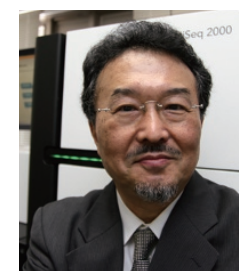

Yuji Kohara, DirectorGeneral, National Institute of Genetics sequencing and large-scale

data analysis. We also have the largest collection of wild-derived strains of mice in Japan," says Kohara. The NIG is also active in building the intellectual infrastructure that ultimately leads to innovation. "Ensuring that the institute maintains its leading position in research is vital," says Kohara. "We invite researchers from all over the world to give seminars and to collaborate on research. We also promote collaboration with industry and other research partners in the Pharma Valley Project. All these activities raise the standard of our basic research."

The new era of genetics research based on highthroughput analyses brings with it the need for massive data handling and storage facilities. In addition to the laboratories, animal breeding and research facilities, experimental farm plots and genetics labs, the NIG campus hosts three buildings dedicated entirely to computer facilities for data storage and manipulation. "We host the DNA Data Bank of Japan - the DDBJ - and are one of three institutes around the world involved in the International Nucleotide Sequence Database Collaboration, which aims to make sequencing data available to everyone," says Kohara. "The information systems supporting life science research form a major aspect of the work conducted at the NIG, and will become even more important in the future."

The activities of research institutions like the NIG and University of Shizuoka are doing much to support the export of Shizuoka's rich health science culture to the world - a local destination with the world at its feet. 

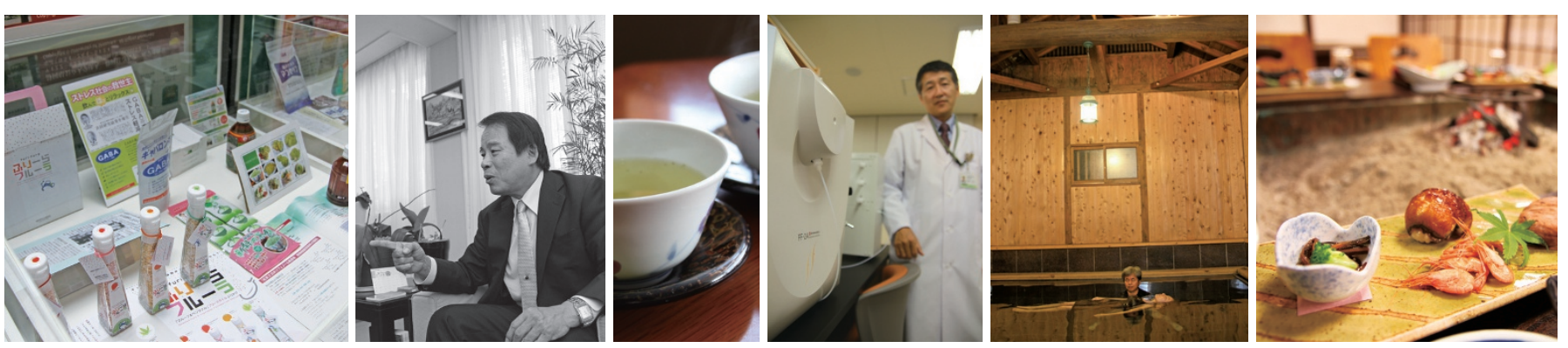

SHIZUOKA REGIONAL RESOURCES

Made in Fuji Country

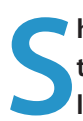

hizuoka is famous in Japan for its green tea and a lifestyle that supports one of the longest average lifespans in the world. Turning that culture of good health and good living into a science-based industry that makes that same lifestyle and longevity available to the world is one of the founding principles of the Pharma Valley Project.

The region around Mount Fuji is blessed with a temperate climate and rich, fertile soil that for centuries have nurtured a strong agricultural industry. Green tea and its health benefits form the foundation for the industry, and nowhere else in the world is there the variety and innovation that can be found among Shizuoka's green tea producers. The region is the largest producer of green tea in Japan, accounting for almost half of total production nationally.

With the 'health revolution' that has swept the world in recent years, it is natural that green tea be the driving force behind the new science-based food industry that has flourished in Shizuoka. "We launched the Food Science Hills project with the prefectural government to turn the regional resources that support our healthy lifestyle, such as green tea, into products with scientifically backed health benefits for consumption by the world," says Naohide Kinae, president of the University of Shizuoka and director of the Food Science Center.

Claims abound as to the purported health benefits of certain foods, and green tea is historically no exception. "That's one of the reasons why myself and my predecessors at the university pushed hard to establish an interdisciplinary School of Food and Nutritional Sciences that brings the latest scientific analyses to nutrition research," says Kinae.

By fostering partnerships between local tea producers, researchers, industry and citizens, the Food Science Hills project is promoting the development of a new generation of green tea beverages and extracts with enhanced health benefits. "We focus on pure tea, and in trying to enhance certain components with known health benefits or improve palatability," says Kinae. "For example, we are producing a white-leaf tea under covers to exclude ultraviolet light. It gives a tea with more of the amino acid theanine, which makes it less bitter and better for markets where there is no culture of green tea consumption." Through modified horticultural and harvesting practices and by introducing technology such as light-emitting diodes to tune polyphenol production, the project is also producing teas with higher content of polyphenols such as catechin and epigallocatechin gallate - a compound now recognized as one of the main health-promoting chemicals in green tea. Some of these teas are already being distributed in Europe. Another aspect of the programme involves investigating how the active compounds in green tea can be made more absorbable and applied as additives to other foods.

The project is not limited to green tea, however. Kinae is in fact a world expert on wasabi horseradish — for which Shizuoka accounts for more than half of all production nationally — and in a collaboration with Masatoshi Kusuhara at the Shizuoka Cancer Center (SCC) is actively working on ways to quantify the claimed anticancer properties of the plant's rhizome and leaves. "It's part of our challenge to bring science to the traditional seasonings of Japan by identifying the molecular mechanisms that we think are the basis for the longevity of Shizuoka's residents," says Kinae.

\section{Healing hot springs}

The Shizuoka prefectural government is keen to ensure that everyone has the opportunity to benefit from the healthcare culture that is now flourishing in the region. Part of that strategy is the Kakaritsuke-yu programme, a network of geothermal spa resorts on Shizuoka's Izu Peninsula that focuses on promoting the physical and spiritual benefits of the Japanese onsen hot spring. "There are many people with good physical health but who suffer from stress," says Yamaguchi. "Through this programme, we want to provide a place where the busy people of Tokyo can come for psychological healing."

The name Kakaritsuke-yu merges the concepts of the 'family doctor'and 'local bath'in a way that invites people to think about onsen resorts as more of a regular place to visit for health improvement than a holiday destination. "The onsen was traditionally curative, but the onsen resorts have since become more like entertainment," says Midori Mizuguchi, coordinator of the Kakaritsuke-yu
Kusuhara uses the latest instruments for metabolome analysis in working to establish a protocol for verifying the health claims of foods. "We are using the millions of dollars worth of equipment at the SCC Research Institute to identify metabolites such as amino acids in order link foods to health effects," he says. "We are perfectly placed for this research, with the best regional produce and a state-of-theart cancer hospital and research institute."

Kusuhara also conducts research into smell and how an understanding of the molecular basis for odour can be used in the clinic. "Using the same instruments as used for food and resource research, we are trying to identify the molecular compounds that produce the distinct odour associated with advanced cancer," says Kusuhara. He has already developed a first-generation anti-odour paste in collaboration with Takasago International. The science of disease odour, now known globally by its Japanese name byoshu, is part of the total care approach to medicine advocated by SCC president Ken Yamaguchi. "We should do whatever we can do ease the burden of cancer, for patients and their families," says Yamaguchi. "Our research at the SCC aims to address all the unmet needs of cancer patients by nurturing a close working relationship between clinicians, researchers and industrial partners."

network. "Through this programme we are getting back to healing."

There are now over 50 onsen in the network each offering a health programme and meet

ing quality and reporting standards for the onsen mineral waters and facilities. A model example of this new type of health spa is Funabarakan, where the services on offer include a deep therapeutic bath and 'water shiatsu', or 'watsu', massage. "I helped develop what is now known as watsu massage while in the US, and the technique is now used all over the world," says Funabarakan owner Motofumi Suzuki. The massage provides a deep meditation-like therapy that, when combined with the resort's unique wasabi hot pot and health-promoting meals, leaves one fully invigorated and ready to once again take on the challenges of city life. 

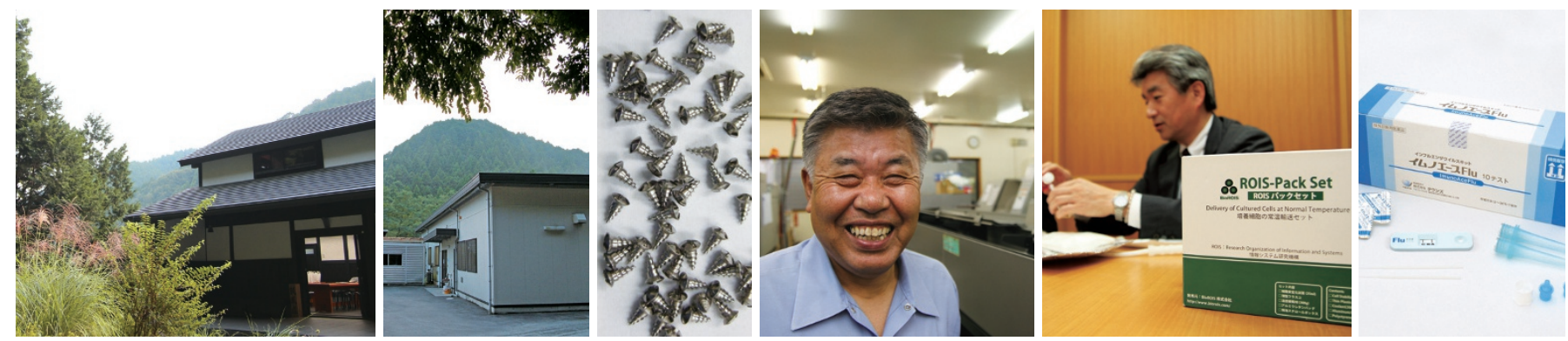

PHARMA VALLEY MANUFACTURERS

\section{Manufacturing a healthy future}

\begin{abstract}
apanese industry has traditionally revolved around heavy manufacturing and the supply of industrial parts, but this is changing with the national government's promotion of growth areas such as health and energy. With the Pharma Valley Project, Shizuoka is leading the country in the shift to a new regional economy with a life science focus.
\end{abstract}

Shizuoka's reinvention as a global exporter of healthrelated technology is well underway, and the region in recent years has claimed the highest output of medical equipment and pharmaceutical products of any region in Japan. The transition from heavy industry such as automobile parts manufacturing to the higher-value market for medical devices, however, has required a sustained effort.

"When we first took some of the clinical needs identified through the Shizuoka Cancer Center to local manufacturers, we found that while they had the technology and expertise, there were major regulatory hurdles to be overcome that local companies had little experience with," says Katsunori Ueda from the Pharma Valley Center (PVC). "One of the major roles of the PVC is in helping local businesses negotiate those regulations."

Shizuoka prefecture provides extensive funding to help local manufacturers arm themselves for entry into the medical device market. "We have an incredible wealth of research capacity and technology in Pharma Valley. The PVC's role is to match needs to local expertise, and then support the manufacturer through the steps required to become a medical devices maker," says Ueda. "One of the main goals of the PVP is to foster a high-value local industry that exports superior medical technology to the world."

This approach to building the capacity and regulatory shrewdness needed for medical device manufacturing has already yielded a series of successes, including a nanoparticle-based influenza test kit developed by TAUNS Laboratories, an automatic high-throughput soft capsule inspection machine produced by Sankyo, and a non-refrigerated cultured cell delivery pack developed in a joint venture led by BioROIS.
But these products are just the start. "Setting up a business for the supply of parts and devices for use in a clinical environment, such as implants or therapeutic devices, requires many years of progressive certification," says Ueda. "The PVP was started in 2001, and only now are we seeing our first local businesses reach the certification levels required to begin producing parts for clinical use - and those parts now need to be submitted for approval by Japan's regulatory agency. It's a long process, but the end result is a profound jump in product value."

One of the local companies that has embarked on this transition from traditional parts manufacturer to medical parts maker is Tokai Buhin Kogyo ('Tokai Parts Manufacturing'). "We started the move to medical eight years ago," says Tokai Buhin president Noboyuki Morita, "and only recently have we achieved the minimum certification required to start producing parts for clinical use."

Tokai Buhin has been manufacturing industrial screws for more than 60 years, predominantly for the auto industry, and over that past two decades Morita has seen technology and the economy change almost beyond recognition. "Cars have changed completely. We started supplying large chassis bolts, and now we manufacture microscrews for computer circuitboards. It's a continuous process of adaptation and renewal, and where we now see a stagnation in auto manufacturing, we see a bright future in medical parts."

Tokai Buhin's technology and expertise in producing microscrews prompted a medical acquaintance to suggest that Morita should look into producing screws for orthopaedic joint fixing. Enamoured with the idea, he paid a visit to the prefectural government for advice. "We had no notion of what might be involved in setting ourselves up for medical manufacturing. Had I known better, perhaps I wouldn't have even considered the idea," laughs Morita. "But in the end, I'm very happy with where we are now."

Morita soon found himself at a governmentfunded incubation centre that provides facilities for developing new products. "At the incubation centre we received amazing support, from both government representatives and other local com- panies, such as Hollyx," says Morita. Hollyx relocated its implant business to Shizuoka in 1997 and is now working closely with the PVC and Shizuoka Cancer Center on developing needs-based devices, such as a diamond-coated bone biopsy kit for minimally invasive diagnosis of bone cancer.

Starting with the introduction of titanium screws in 2003, Tokai took out ISO 9001 certification in 2005, which allowed the company to start producing certain types of medical devices. That was followed by the ISO 13485 certification required to start manufacturing parts for clinical use. "The process and technology is the accumulation of many steps. We had to up-train our staff at each stage, upgrade our equipment and facilities, formalize and refine our processes, and implement quality assurance in every aspect of our work. It was hard, but now of course we have the best, multifunctional staff, quality facilities and the latest technology, and we're producing parts that truly help improve lives and society," says Morita.

A close working relationship with the PVP's regulatory agent, Fuji Pharma, has helped streamline the process of regulation for locally manufactured medical devices. By being one of the first companies to make the transition to certified medical device manufacturer, Tokai Buhin itself also plays a pivotal role in helping other local companies gain a foothold in the sector. "Some of our plastic prod ucts, for example," says Morita, "are manufactured by other local businesses, but final assembly and quality assurance are performed here in order to meet regulatory requirements."

Tokai Buhin is now manufacturing titanium screws for fixing joints and skull plates - the technology for which took two years to develop. The company is now one of the top manufacturers of skull screws, and some of the microscopic refinements Tokai has introduced make their microscrews highly favoured by clinicians. Setting the company apart from the crowd, too, is its 'Fuji spirit', illustrated most aptly by Morita's decision to base the medical devices factory in the forested hills of eastern Shizuoka. "We are inspired and taught by nature to seek a fusion of traditional craftsmanship with technology in a way that contributes to health and well-being." 


\section{Contact details}
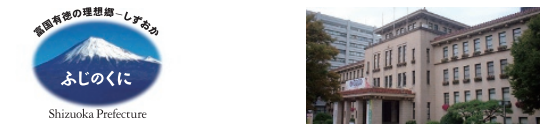

Shizuoka Prefecture

9-6 Otemachi, Aoi, Shizuoka

Shizuoka 420-8601, Japan

www.pref.shizuoka.jp/a_foreign/english

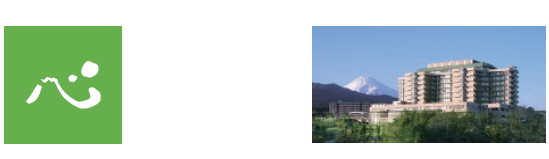

Shizuoka Cancer Center

1007 Shimonagakubo, Nagaizumi,

Sunto, Shizuoka 411-8777, Japan

Tel: +81-55-989-5222

Email: info@scchr.jp

www.scchr.jp/english

\section{1}

Ziji PharmaValley

Pharma Valley Center

1007 Shimonagakubo, Nagaizumi,

Sunto, Shizuoka 411-8777, Japan

Tel: +81-55-980-6333

Email: mail@fuji-pvc.jp

www.fuji-pvc.jp/english

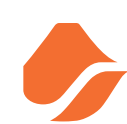

University of Shizuoka

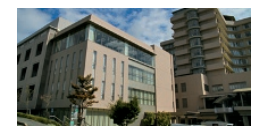

52-1 Yada, Suruga, Shizuoka,

Shizuoka 422-8526, Japan

Tel: +81-54-264-5124

Email: inquiry@u-shizuoka-ken.ac.jp

http://eng.u-shizuoka-ken.ac.jp/

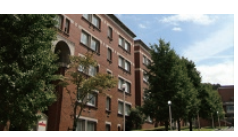

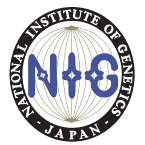

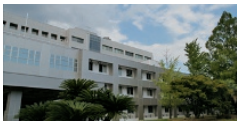

National Institute of Genetics

1111 Yata, Mishima, Shizuoka 11-8540, Japan

Email: infokoho@nig.ac.jp

www.nig.ac.jp/index-e.htm

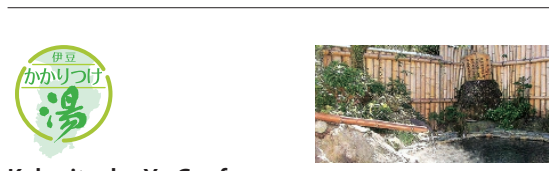

Kakaritsuke-Yu Conference

2-31-145 Bunkyo, Mishima

Shizuoka 411-8555, Japan

Tel: +81-55-980-0783

Email: kanken@ir.nihon-u.ac.jp

www.kakaritsukeyu.jp/english/index.aspx
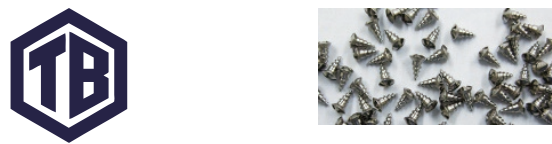

Tokai Buhin Kogyo Co. Ltd

9-11-12 Futaba, Numazu, Shizuoka 410-0005, Japan Tel: +81-55-921-4174

Email: infotokabuko@tokaibuhin.co.jp

www.tokaibuhin.co.jp/en/

\section{(協) Sankyo Co., Ltd.}

Sankyo Co. Ltd

573-13 Denbo, Fuji, Shizuoka 417-0061, Japan

Tel: +81-545-22-2558

Email: info@sankyocoltd.co.jp

www.sankyocoltd.co.jp/english.htm
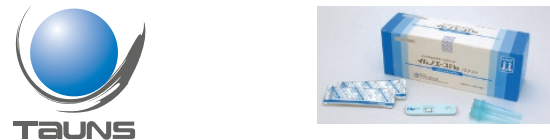

TAUNS Laboratories Inc

9-12-2 Futaba, Numazu, Shizuoka 410-0005, Japan Tel: +81-55-925-6200

Email: kubodera@tauns.co.jp

www.tauns.co.jp/english

\section{$\theta$ HOLLYX}

Hollyx Co. Ltd

294-46 Ashitaka, Numazu,

Shizuoka 410-0001, Japan

Tel: +81-55-925-4601

Email: horiuchi@hollyx.co.jp

www.hollyx.co.jp/english/index_e.html

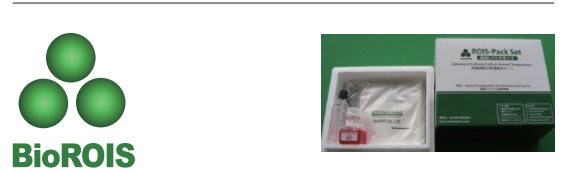

BioROIS

\section{BioROIS Co. Ltd}

Mishima-Suruga Building, 15-26 Ichiban

Mishima, Shizuoka 411-0036, Japan

Tel: +81-55-994-9855

Email: info@biorois.com

www.biorois.com/eng/

\section{P \\ Fuji Pharma

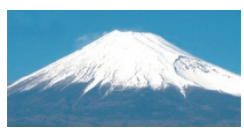

Fuji Pharma Co. Ltc

9-8-31 Imaizumi, Fuji, Shizuoka 417-0001, Japan

Tel: +81-545-55-5210

Email:world_info@fujipharma.co.jp

www.fuji-fujipharma.jp

\section{Shizuoka from the sky (view from the north)}

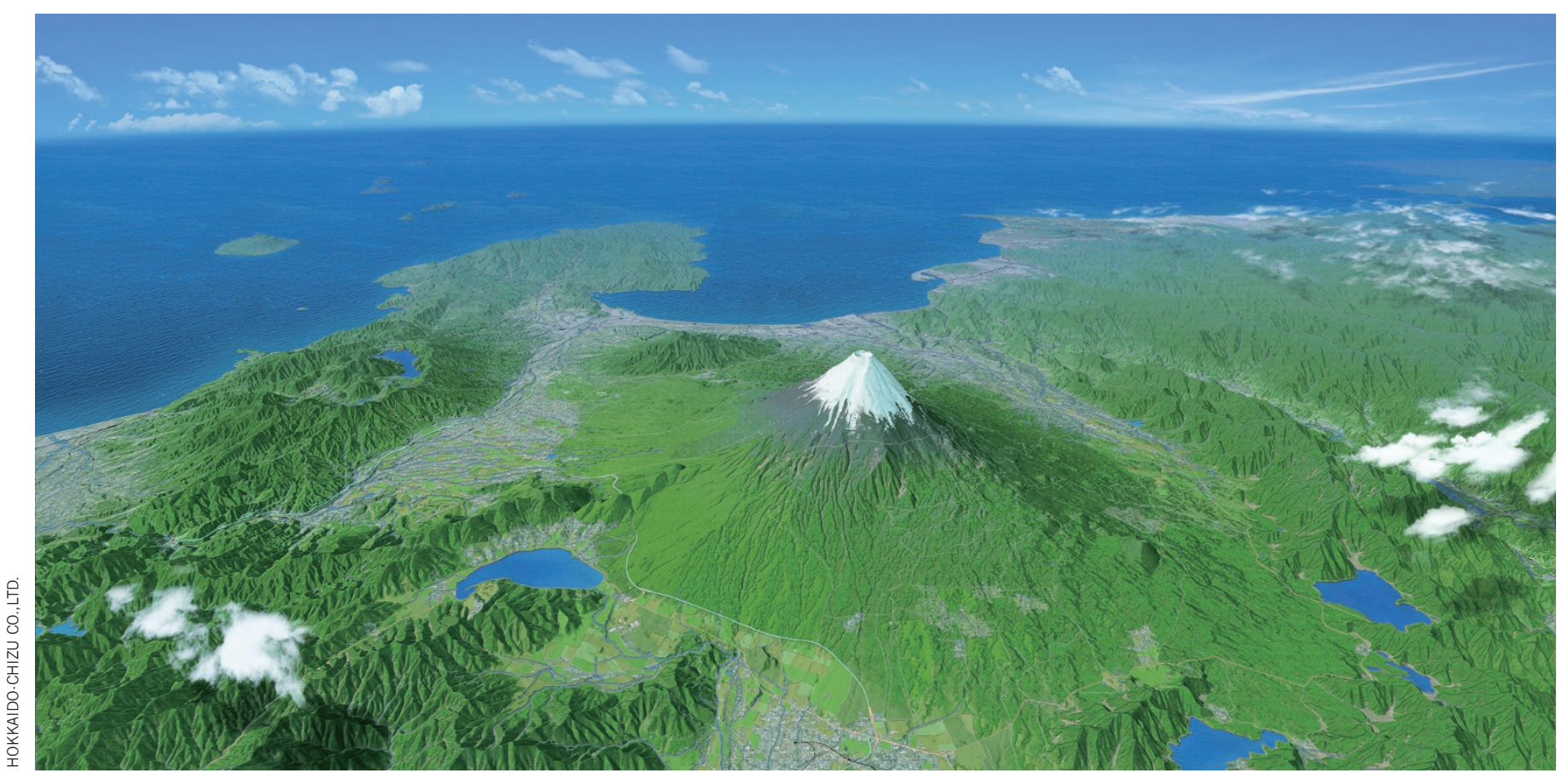

